\title{
EFECTOS DE UN PROGRAMA DE ACTIVIDAD FÍSICA CON EL MÉTODO PILATES SOBRE LA FUNCIONALIDAD DE MUJERES EMBARAZADAS. ESTUDIO PILOTO
}

\section{EFFECTS OF A PILATES PHYSICAL ACTIVITY PROGRAM ON THE FUNCTIONALITY OF PREGNANT WOMEN. PILOT STUDY}

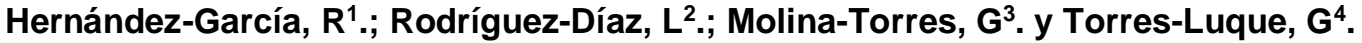 \\ ${ }^{1}$ Hernández-García, R. Facultad de Ciencias del Deporte. Universidad de Murcia (España). \\ rhernandezgarcia@um.es \\ ${ }^{2}$ Rodríguez-Díaz, L. Matrón. Hospital Universitario de Ceuta. lucianin000@gmail.com \\ ${ }^{3}$ Molina-Torres, G. Facultad de Humanidades y Ciencias de la Educación. Universidad de Jaén \\ (España).gmolina@ujaen.es \\ ${ }^{4}$ Torres-Luque, G. Facultad de Humanidades y Ciencias de la Educación. Universidad de Jaén \\ (España).gtluque@ujaen.es
}

Código UNESCO: 5899 Otras Especialidades Pedagógicas.

Clasificación Consejo de Europa: 14. Fisioterapia y rehabilitación.

Recibido el 13 de marzo de 2018

Aceptado el 11 de mayo de 2018

Correspondencia:

Gema Torres-Luque

gtluque@ujaen.es

DOI: http://dx.doi.org/10.24310/riccafd.2018.v7i2.5088

\section{RESUMEN}

El presente estudio pretende determinar el efecto de un programa de Pilates sobre la funcionalidad en mujeres gestantes y analizar su repercusión en el parto. Un grupo de 10 mujeres embarazadas desarrollaron un programa de Pilates durante 8 semanas (2 sesiones/semana), se valoró la funcionalidad global a través de los test funcionales Overhead Squat (OHS), Hurdle Step (HS), Forward Step Down (FSD); Shoulder Mobility (SM) y Active Straight Leg Raise (ASLR), así como el tipo de parto posterior. Los resultados muestran una mejora de la funcionalidad del Hurdle test, Forward step down y Shoulder mobility test $(p<0,01)$, así como un descenso significativo de las compensaciones y riesgo de lesión global después del programa de actividad física $(p<0,01)$. A su vez, se tuvo un parto más saludable. Los efectos del Pilates en mujeres embarazadas, ofrecen mejoras en la funcionalidad global y un efecto positivo en el parto.

PALABRAS CLAVE: pilates, embarazo, funcional, lesiones, parto 


\section{ABSTRACT}

This study aims to determine the effect of a Pilates programme on functionality in pregnant women; and to analyse its impact on childbirth. A group of 10 pregnant women developed a Pilates program for 8 weeks (2 sessions/week), overall functionality was assessed through functional tests Overhead Squat (OHS), Hurdle Step (HS), Forward Step Down (FSD), Shoulder Mobility (SM) and Active Straight Leg Raise (ASLR), as well as the type of subsequent delivery. The results show an improvement in the functionality of the Hurdle test; Forward step down and Shoulder mobility test $(p<0.01)$, as well as a significant decrease in compensation and overall risk of injury after the physical activity programme $(p<0.01)$. In turn, the delivery was healthier. The effects of Pilates on pregnant women offer improvements in overall functionality and a positive effect on childbirth.

KEY WORDS: Pilates, pregnancy, functional, injuries, childbirth

\section{INTRODUCCIÓN}

El embarazo es un periodo momentáneo que suele tener una duración de entre 38 y 40 semanas ${ }^{(1)}$. A pesar de que el embarazo se asocia con cambios desde el punto de vista morfológico y funcional, en realidad, los beneficios de realizar actividad física son indiscutibles $\left({ }^{2}\right)$. La literatura científica muestra como actividad física de carácter aeróbico es la más desarrollada en embarazadas ${ }^{(3-}$ 6), aunque en los últimos tiempos el ejercicio con un carácter de fuerza también aparece ${ }^{(7,8)}$.

Dentro del carácter de fuerza, los ejercicios más recomendados son aquellos que colaboran con la musculatura involucrada en el parto y los músculos que soportan el peso corporal y mantienen la postura ${ }^{(9)}$. Es por ello, que una de las actividades seleccionadas en el periodo de gestación sea el método Pilates, el cual ha tenido un incremento exponencial en esta población $(10,11)$. Este método, dirigido a las gestantes pretende mejorar la armonía muscular, control postural e higiene, sin forzar al individuo (11). Incluye actividades adaptadas a la nueva situación de la mujer, pudiendo llevarse a cabo en cualquier momento a lo largo de la gestación, donde no es necesario una experiencia previa en el mismo para poder ser desarrollado ${ }^{(12,13)}$.

Se ha constatado como el incremento de la masa corporal que se produce a lo largo del embarazo puede desencadenar en dolor a nivel lumbar, aspecto que sufren en torno al $20-90 \%$ de mujeres embarazadas ${ }^{(14,15)}$. Este dolor puede afectar a la habilidad de sentarse, levantarse, caminar, es decir, no desarrollar una vida cotidiana con normalidad (16). Tener por ejemplo, un dolor en la zona baja de la espalda, parece cambiar el patrón de movimiento ${ }^{(17)}$. Los patrones de movimiento (PM) son secuencias motoras programadas innatas a nivel del sistema nervioso central (SNC) que definen la base de cada movimiento en el ser humano. Debido al tipo de desarrollo de cada individuo en sus etapas tempranas, o por etapas de dolor crónico (como durante el embarazo) los PM 
pueden verse afectados y fijados en el SNC con una errónea calidad de movimiento. Por lo tanto, disponer de PM erróneos en conductas motoras puede incrementar el riesgo de dolor y lesión, debido a desequilibrios musculares y articulares. Es decir, si el movimiento se realiza sin calidad, se expone a los tejidos a una carga excesiva que eleva el riesgo de lesiones y disminuyendo la funcionalidad del movimiento (18). Por tanto, el concepto de calidad de movimiento, se postula como un predictor del riesgo de dolor y futuras lesiones (19). En este sentido, y considerando que la actividad física de Pilates puede mejorar la funcionalidad en sus practicantes ${ }^{(20)}$ se intuye que estos beneficios también podrían repercutir en la población gestante. Bajo nuestro conocimiento no se ha analizado, en la literatura científica consultada, ningún estudio que analice el efecto del método Pilates sobre el perfil funcional en mujeres embarazadas.

Por lo tanto, los objetivos del presente estudio son: a) Determinar el efecto de un programa de actividad física de 8 semanas de duración por medio del método Pilates sobre la funcionalidad global en mujeres gestantes; y b) Analizar el efecto diferido en el parto de este grupo poblacional.

\section{MATERIAL Y METODO}

\section{Muestra}

La muestra estuvo compuesta por 10 mujeres embarazadas $(30,80 \pm 3,99$ años; $1,61 \pm 0,81 \mathrm{~m} ; 66,44 \pm 8,48 \mathrm{~kg}$ ). Como criterios de inclusión se consideró: a) Ser mayor de edad; b) Estar en el segundo trimestre de embarazo; c) Que no sea embarazo múltiple; d) No tener ningún tipo de contraindicación de carácter médico ni patologías; e) No tomar ningún tipo de medicamento que pueda influir en el programa; f) No participar en otros programas de ejercicio físico; g) No tener ninguna contraindicación o lesión que le impida la realización de actividad física. Todas las embarazadas firmaron un consentimiento escrito para participar en el estudio siguiendo las indicaciones de la Declaración de Helsinki.

\section{Procedimiento}

Se llevó a cabo una valoración inicial, un programa de actividad física y una valoración final.

La valoración inicial (pre-test) estuvo compuesta por 5 test funcionales para evaluar los patrones de movimiento globales: Overhead Squat (OHS), Hurdle Step (HS), Forward Step Down (FSD), Shoulder Mobility (SM) y Active Strength Leg Raise (ASLR).

Overhead Squat (OHS): Fue administrado según las instrucciones de ${ }^{(21)}$. La sujeto asume la posición inicial colocando sus pies aproximadamente a la altura de los hombros y los pies alineados en el plano sagital. Además eleva sus brazos hacia arriba con los hombros flexionados y abducidos, y los codos extendidos. A continuación, se indica al individuo que descienda hasta donde 
pueda, sin perder la posición de los brazos. Después vuelve a la posición inicial. Se realizaron tres repeticiones. No existe imagen visual, sólo orden verbal.

Hurdle Step Test (HS): Fue administrado según las instrucciones de ${ }^{(21)}$, modificando la posición de los brazos, los cuales se pondrán en el pecho entre cruzados. El sujeto asume la posición inicial colocando los pies juntos. A continuación, se le pide que mantenga una postura erguida, que levante una rodilla (flexión de cadera y rodilla) todo lo que pueda manteniendo la postura. La pierna en movimiento es entonces devuelta a la posición inicial. Se realizaron tres repeticiones con cada pierna. No existe imagen visual, sólo orden verbal.

Forward step down (FSD): Se desarrolló según (22). Las participantes estaban de pie en una pierna en una caja de $20 \mathrm{~cm}$ de altura, con los brazos cruzados sobre el pecho. A continuación, se les pidió que se agacharan lo más lejos posible doblando la rodilla en la pierna que soportaba el peso hasta que el talón de la otra extremidad tocó el suelo, manteniendo su equilibrio y volviendo a la posición inicial. Se realizaron tres repeticiones con cada pierna. No existe imagen visual, sólo verbal.

Shoulder mobility test (SM): Se desarrolló según el protocolo de ${ }^{(21)}$. Las participantes se colocaron de pie, con los brazos en cruz; y a la instrucción del evaluador se le pide que eleve un brazo por encima de la región torácica mientras el otro brazo por debajo, intentando colocar sus manos por detrás de la espalda, cerradas en puños, tan cerca entre ellas como sea posible. Se realizaron tres repeticiones por cada lado. Existe una demostración previa del movimiento por parte del evaluador.

Active Straight Leg Raise (ASLR): Se desarrolló según el protocolo que marcan (23). Las participantes se tumban en supino, con piernas estiradas y separadas a la anchura de las caderas, y cuando reciban la indicación, elevan una pierna estirada todo lo que puedan. Después vuelven a la posición inicial. Se realizaron tres repeticiones con cada pierna. No existe demostración, sólo indicaciones verbales.

Cada test fue grabado usando tres cámaras para cubrir los planos frontal anterior, posterior y el plano sagital para cada una de las repeticiones. La cámara de video se colocó aproximadamente a $3 \mathrm{~m}$ de cada participante y a una altura de $1,5 \mathrm{~m}$. Los datos de vídeo se transfirieron a un ordenador personal. Cada ensayo se revisó para asegurar un punto de inicio consistente para su visualización y luego se guardó para su evaluación. Todas las visualizaciones fueron supervisadas por el mismo evaluador certificado por el mismo especialista en valoración funcional con 4 años de experiencia. La tabla 1 muestra los puntos de valoración de cada uno de los test y su traducción a las compensaciones a los que pueden derivar. 
Tabla 1. Puntuación conjunta de los test de valoración de la calidad de movimiento.

$\begin{array}{cc}\text { Valoración Ejecución } & \text { Compensaciones } \\ \text { (1 punto cada ítem }) & (1 \text { punto cada ítem })\end{array}$

\begin{tabular}{|c|c|c|}
\hline $\begin{array}{l}\text { Overhead } \\
\text { Squat (OHS) }\end{array}$ & $\begin{array}{l}\text { - Ejecuta la triple flexión- } \\
\text { extensión de cadera, rodilla y } \\
\text { tobillo. } \\
\text { - Tórax paralelo a la tibia o } \\
\text { vertical al suelo. } \\
\text { - Fémur por debajo de la } \\
\text { horizontal de la rótula. } \\
\text { - Rodillas alineadas con los pies } \\
\text { (izquierdo y derecho) }\end{array}$ & $\begin{array}{l}\text { - Exceso de lordosis lumbar. } \\
\text { - Pérdida de disociación lumbo-pélvica. } \\
\text { - Exceso de cifosis } \\
\text { - Brazos caen al frente. } \\
\text { - Los pies giran hacia fuera (izquierdo y } \\
\text { derecho) } \\
\text { - Rodillas en valgo (izquierda y } \\
\text { derecha) } \\
\text { - Rodillas en varo (izquierda y derecha) } \\
\text { - Inversión de la subastragalina } \\
\text { (izquierda y derecha) } \\
\text { - Eversión de la subastragalina } \\
\text { (izquierda y derecha) } \\
\text { - Elevación de talones (izquierda y } \\
\text { derecha) } \\
\text { - Dismetría en la carga (hacia } \\
\text { izauierda o derecha) }\end{array}$ \\
\hline
\end{tabular}

Total OHS Ejecución: de 0 a 5 puntos

Compensaciones: de 0 a 13

\begin{tabular}{|c|c|c|}
\hline $\begin{array}{l}\text { Hurdle } \\
\text { (HS) }\end{array}$ & $\begin{array}{l}\text { Observar en ambas } \\
\text { extremidades: } \\
\text { - Ejecuta la triple flexión de } \\
\text { cadera, rodilla y tobillo. } \\
\text { - Alineación cadera, rodilla y } \\
\text { tobillo. } \\
\text { - Mínimo movimiento torácico. } \\
\text { - Pelvis estable. }\end{array}$ & $\begin{array}{l}\text { Observar en ambas extremidades: } \\
\text { - Exceso de lordosis lumbar. } \\
\text { - Pérdida de disociación lumbo-pélvica. } \\
\text { - Exceso de cifosis } \\
\text { - Rodillas en valgo. } \\
\text { - Rodillas en varo. } \\
\text { - Inversión de la subastragalina. } \\
\text { - Eversión de la subastragalina. } \\
\text { - Elevación de talones. } \\
\text { - Rotación externa/interna pie apoyo } \\
\text { - Rotación externa/interna pierna flexión } \\
\text { - Basculación/rotación pélvica }\end{array}$ \\
\hline Total HS & Ejecución: de 0 a 8 puntos & $\begin{array}{c}\text { Compensaciones: de } 0 \text { a } 22 \text { (ambas } \\
\text { piernas) }\end{array}$ \\
\hline $\begin{array}{l}\text { Forward step } \\
\text { down (FSD) }\end{array}$ & $\begin{array}{l}\text { Observar en ambas } \\
\text { extremidades: } \\
\text { - Ejecuta la triple flexión de } \\
\text { cadera, rodilla y tobillo. } \\
\text { - Alineación cadera, rodilla y } \\
\text { tobillo. } \\
\text { - Mínimo movimiento torácico. } \\
\text { - Pelvis estable. }\end{array}$ & $\begin{array}{l}\text { Observar en ambas extremidades: } \\
\text { - Exceso de lordosis lumbar. } \\
\text { - Pérdida de disociación lumbo-pélvica. } \\
\text { - Exceso de cifosis } \\
\text { - Rodillas en valgo. } \\
\text { - Rodillas en varo. } \\
\text { - Inversión de la subastragalina. } \\
\text { - Eversión de la subastragalina. } \\
\text { - Elevación de talones. } \\
\text { - Rotación externa/interna pie apoyo } \\
\text { - Rotación externa/interna pierna flexión } \\
\text { - Basculación/rotación pélvica }\end{array}$ \\
\hline Total FSD & Ejecución: de 0 a 8 puntos & $\begin{array}{c}\text { Compensaciones: de } 0 \text { a } 22 \text { (ambas } \\
\text { piernas) }\end{array}$ \\
\hline
\end{tabular}




\begin{tabular}{|c|c|c|}
\hline $\begin{array}{l}\text { Shoulder } \\
\text { mobility test } \\
\text { (SM) }\end{array}$ & $\begin{array}{l}\text { Observar en ambas } \\
\text { extremidades: } \\
\text { - Distancia menos de una palma } \\
\text { de la mano } \\
\text { - Mínimo movimiento torácico } \\
\text { - Codos alineados en el plano } \\
\text { frontal } \\
\text { - Mínimo movimiento cervical }\end{array}$ & $\begin{array}{l}\text { Observar en ambas extremidades: } \\
\text { - Existencia de protusión de la cabeza. } \\
\text { - Aparece lordosis lumbar. } \\
\text { - Escápula alada. }\end{array}$ \\
\hline Total SM & Ejecución: de 0 a 8 puntos & $\begin{array}{c}\text { Compensaciones: de } 0 \text { a } 6 \text { (ambos } \\
\text { brazos) }\end{array}$ \\
\hline $\begin{array}{l}\text { Active Straight } \\
\text { Leg Raise } \\
\text { (ASLR) }\end{array}$ & $\begin{array}{l}\text { Observar en ambas } \\
\text { extremidades: } \\
\text { - Supera la pierna totalmente } \\
\text { extendida el punto medio del } \\
\text { fémur } \\
\text { - Supera la pierna totalmente } \\
\text { extendida talón el punto de rótula }\end{array}$ & $\begin{array}{l}\text { Observar en ambas extremidades: } \\
\text { - La pelvis se muestra inestable. } \\
\text { - La pierna de apoyo se modifica } \\
\text { durante el movimiento. } \\
\text { - Rotación externa/interna pie apoyo } \\
\text { - El cuello es inestable. } \\
\text { - El lumbar es inestable. } \\
\text { - Los hombros son inestables. }\end{array}$ \\
\hline Total ASLR & Ejecución: de 0 a 2 punto & $\begin{array}{c}\text { Compensaciones: de } 0 \text { a } 12 \text { (ambas } \\
\text { piernas) }\end{array}$ \\
\hline $\begin{array}{l}\text { TOTAL (suma } \\
\text { todos los test) }\end{array}$ & Ejecución: 0 a 27 puntos & Compensaciones: 0 a 75 puntos \\
\hline
\end{tabular}

Además se calculó el \% de riesgo de lesión global, en función del número de compensación total (sumatorio de las compensaciones de cada test), a través de la fórmula que a continuación se describe:

\% Riesgo lesión global $=$ ( $n$ o compensaciones OHS $+n^{\circ}$ compensaciones $\mathrm{HT}+\mathrm{n}^{\circ}$ compensaciones FSD $+\mathrm{n}^{\circ}$ compensaciones $\mathrm{SM}+\mathrm{n}^{\circ}$ compensaciones ASLR) $\times 100 / 75$

Programa de actividad física por el método Pilates:

Se realizó un programa de actividad física por medio del método Pilates durante un tiempo de 8 semanas, dos veces por semana, con una duración de la sesión entre 40 y 45 minutos. Todas las sesiones se estructuraron de la misma manera: una parte de calentamiento (5-8 $\mathrm{min})$, parte de tonificación por medio de posturas de Pilates específico para embarazadas (25-30 min); ejercicios de flexibilidad (5-10 min) y ejercicios de respiración (5 min) ${ }^{(24)}$.

Después del programa de intervención se realizó una valoración final (post-test) con las mismas pruebas y el mismo protocolo que la valoración inicial.

Posteriormente, se realizó el seguimiento del embarazo y la finalización del mismo (parto), donde se controlaron las siguientes variables: a) tipo de parto (parto eutócico, parto distósico y parto por cesárea) como resultado final (25); b) Realización de episiotomía (si o no); c) Inducción del parto (si o no); d) Motivo 
de la inducción (no hay inducción, motivo justificado, motivo injustificado); e) Empleo de analgesia epidural (si o no) y, d) Centímetros de dilatación.

\section{Análisis estadístico}

Se empleó el paquete estadístico SPSS versión 20.0 para Windows. Se realizó un análisis descriptivo de los datos mostrados como media y desviación típica; así como de frecuencias para el análisis del tipo de parto. Se empleó la prueba $\mathrm{T}$ Student para muestras relacionadas para observar las posibles diferencias entre antes y después del programa de actividad física. La significación se fijó en $\mathrm{p}<0.05$.

\section{RESULTADOS}

En la tabla 2 se muestran los cambios producidos en la calidad de movimiento en las gestantes objeto de estudio antes (pre-test) y después (posttest) del programa de intervención con el método Pilates de ocho semanas de duración. 
Tabla 2. Cambios producidos antes y después del programa de actividad física

\begin{tabular}{|c|c|c|c|c|}
\hline & Pre-test & Post-test & $P$ & Cambio \\
\hline Masa (kg) & $76,58 \pm 12,14$ & $78,12 \pm 11,46$ & $P=0,934$ & $\uparrow$ \\
\hline Talla (cm) & $1,63 \pm 0,12$ & $1,63 \pm 0,10$ & $P=0,000$ & $=$ \\
\hline $\mathrm{IMC}\left(\mathrm{kg} / \mathrm{m}^{2}\right)$ & $25,50 \pm 2,06$ & $26,19 \pm 2,01$ & $P=0,000$ & $\uparrow$ \\
\hline OHS Ejecución & $1,80 \pm 1,13$ & $1,30 \pm 0,48$ & $P=0,244$ & $\downarrow$ \\
\hline $\begin{array}{l}\text { OHS } \\
\text { Compensaciones }\end{array}$ & $6,50 \pm 1,08$ & $5,50 \pm 1,35$ & $P=0,096$ & $\downarrow$ \\
\hline HS Ejecución & $1,85 \pm 1,20$ & $2,00 \pm 0,94$ & $P=0,769$ & $\uparrow$ \\
\hline $\begin{array}{l}\text { HS } \\
\text { Compensaciones }\end{array}$ & $3,70 \pm 0,94$ & $2,10 \pm 0,73$ & $P=0,006$ & $\downarrow$ \\
\hline FSD Ejecución & $1,65 \pm 0,88$ & $1,90 \pm 0,73$ & $P=0,551$ & $\uparrow$ \\
\hline $\begin{array}{l}\text { FSD } \\
\text { Compensaciones }\end{array}$ & $4,05 \pm 1,06$ & $2,30 \pm 0,82$ & $P=0,007$ & $\downarrow$ \\
\hline SM Ejecución & $0,65 \pm 0,66$ & $1,30 \pm 0,23$ & $P=0,169$ & $\uparrow$ \\
\hline $\begin{array}{l}\text { SM } \\
\text { Compensaciones }\end{array}$ & $1,90 \pm 0,31$ & $1,30 \pm 0,48$ & $P=0,005$ & $\downarrow$ \\
\hline ASLR Ejecución & $1,20 \pm 0,42$ & $1,60 \pm 0,51$ & $P=0,104$ & $\uparrow$ \\
\hline $\begin{array}{l}\text { ASLR } \\
\text { Compensaciones }\end{array}$ & $2,55 \pm 1,06$ & $2,30 \pm 0,48$ & $P=0,537$ & $\downarrow$ \\
\hline $\begin{array}{l}\text { Compensaciones } \\
\text { totales }\end{array}$ & $18,70 \pm 2,58$ & $13,50 \pm 2,41$ & $P=0,003$ & $\downarrow$ \\
\hline \% Riesgo global & $24,93 \pm 5,49$ & $18,72 \pm 5,13$ & $P=0,003$ & $\downarrow$ \\
\hline
\end{tabular}

OHS: Overhead squat; HS: Hurdle test; FSD: Forward step down; SM: Shoulder mobility test; ASLR: Active straight leg raise. 
En cuanto a la calidad de movimiento, se observa un descenso en las compensaciones del Hurdle test $\left(\mathrm{t}_{(9)}=3,539, \mathrm{p}<0,01\right)$; Forward step down $\left(\mathrm{t}_{(9)}=\right.$ $3,490, p<0,01)$ y Shoulder mobility test $\left(t_{(g)}=3,674, p<0,01\right)$. Las compensaciones totales descienden después del programa de actividad física $\left(t_{(9)}=4,117\right.$, $p<0,01)$, así como el riesgo de lesión global $\left(t_{(9)}=4,118, p<0,01\right)$, por lo tanto, aparece una mejora de la funcionalidad.

En la tabla 3 se muestran los datos referentes al proceso de parto en las gestantes.

Tabla 3. Parámetros obstétricos de finalización del proceso del parto.

\begin{tabular}{|c|c|c|c|}
\hline & & Frecuencia & Porcentaje \\
\hline \multirow{3}{*}{ Tipo de embarazo } & Eutósico & 10 & 100 \\
\hline & Distócico & 0 & 0 \\
\hline & Cesárea & 0 & 0 \\
\hline \multirow{2}{*}{ Episiotomía } & No hay & 0 & 100 \\
\hline & Si hay & 0 & 0 \\
\hline \multirow[b]{2}{*}{ Inducción } & $\mathrm{Si}$ & 1 & 10 \\
\hline & No & 9 & 90 \\
\hline \multirow{3}{*}{ Motivo Inducción } & No hay & 9 & 90 \\
\hline & Motivo Justificado & 1 & 10 \\
\hline & Motivo injustificado & 0 & 0 \\
\hline \multirow{2}{*}{ Analgesia } & $\mathrm{Si}$ & 5 & 50 \\
\hline & No & 5 & 50 \\
\hline \multirow{3}{*}{ Analgesia $(\mathrm{cm})$} & $0-3$ & 5 & 50 \\
\hline & $3-6$ & 1 & 25 \\
\hline & $6-10$ & 1 & 25 \\
\hline
\end{tabular}

Se aprecia como la muestra ha tenido un parto normal en su totalidad, así como no ha sido necesario episiotomía en ningún caso. Solo existió un caso justificado de inducción y el $50 \%$ de la muestra no necesitó ningún tipo de analgesia. 


\section{DISCUSIÓN}

Una de las fortalezas de este estudio es haber realizado un programa de intervención por medio del método Pilates para ver el efecto sobre la funcionalidad y sobre el parto en un grupo poblacional como son las gestantes.

A nivel de calidad de movimiento, el desarrollo de 8 semanas de un programa con el método Pilates en mujeres embarazadas, ha provocado cambios significativos en los test funcionales utilizados, especialmente en los de apoyo monopodal (HS y FSD) y en el test de movilidad de hombros (SM). Tras las 16 sesiones de Pilates, las gestantes han disminuido significativamente el número de compensaciones en el HS $(p=0,006)$ y en el FSD $(p=0,007)$. El hecho de disminuir el número de compensaciones en cada test, puede suponer una reducción del riesgo de dolor crónico o posible estado lesivo, por lo que conlleva una posible mejora de su funcionalidad, y por lo tanto de su calidad de vida. Tanto es así, que los resultados del HS y FSD están íntimamente relacionados entre si, ya que ambos recogen información sobre la movilidad, estabilidad, control motor y simetría de una pierna. Indicando que las mujeres embarazadas de este estudio, han mejorado la base funcional de los patrones de movimiento de marcha (coordinación alternativa de flexión-extensión de cadera), y de subida-bajada del escalón (empuje sobre una pierna). Estos datos coinciden con los mostrados por ${ }^{(26)}$ que indican mejoras significativas de fuerza y resistencia en las extremidades inferiores de mujeres tras su participación en un programa de pilates, comparadas con mujeres que llevan su vida normal. Aparecen recomendaciones donde (27) manifiestan la necesidad de establecer planes de prevención para evitar las caídas en mujeres embarazadas, de hecho incluyen actividades dirigidas como el yoga o el pilates. Los resultados del presente estudio indican que el Pilates puede mejorar la estabilidad y la fuerza a una pierna de las mujeres gestantes.

Respecto a las mejoras en el SM, las mujeres del estudio muestran un descenso significativo del número de compensaciones $(p=0,005)$ en el raquis tanto cervical como lumbar. Esto indica que el desarrollo del programa de Pilates durante 8 semanas, parece que ha influido en una mejora de estabilidad de la columna vertebral mientras existe máxima movilidad del hombro (rotación externa más abducción, así como rotación interna más aducción). Fourie y colaboradores ${ }^{(28)}$ muestran mejoras en la flexibilidad de hombros en mujeres con un programa de 8 semanas de Pilates Mat, especialmente en la flexión de hombro. Sin embargo, no se han encontrado referencias con mujeres embarazadas. Así como en un estudio de Cruz-Ferreira muestran una mejora de en la alineación frontal y sagital de los hombros, tras un programa de 6 meses de Pilates con mujeres de mediana edad (29). Teniendo en cuenta que el Pilates incorpora ejercicios de estiramientos suaves y regulares, donde la longitud del tejido conectivo puede mejorar, así como contribuir al alargamiento de las fibras contráctiles $(28,30)$ parece lógico que las mujeres embarazadas mejorasen los valores obtenidos en el $\mathrm{SH}$. 
En cuanto al resto de test como el OHS, muestra valores de ejecución menores en la valoración final, con respecto a la inicial. Sin embargo, el número de compensaciones mostradas, es mejor en la valoración final (tabla 2). Esto se puede deber al incremento del peso corporal de las gestantes, las cuales pueden verse afectadas al realizar la triple flexión-extensión de tobillo, rodilla y cadera con mayor amplitud ${ }^{(16)}$. En cambio, a pesar de no bajar mucho el cuerpo, lo han hecho con un menor número de compensaciones, por lo que la calidad de movimiento ha mejorado, a pesar de no ser cambios estadísticamente significativos $(p=0,096)$.

En cuanto al ASLR, las puntuaciones de ejecución han mejorado sin llegar a ser significativas $(p=0,104)$, considerando que los valores obtenidos en cuanto al número de compensaciones en este test es menor tras el programa de Pilates, sin llegar a ser significativo $(p=0,537)$. Estos datos son similares a los encontrados en otros estudios donde las mujeres tras diferentes programas por medio el método Pilates incrementaban los valores obtenidos tanto en flexión como en extensión de cadera ${ }^{(28,29)}$. En el presente estudio, no sólo mejoran la ejecución, sino que disminuyen el número de compensaciones, asegurando una mayor calidad de movimiento.

A su vez, es necesario destacar que como este grupo de test ofrecen la posibilidad de analizar el riesgo global de lesión, el mismo, en la muestra objeto de estudio, ha disminuido $(\mathrm{p}=0,003)$. Si la calidad de movimiento mejora, mejora la funcionalidad, y a pesar del incremento del peso corporal, el riesgo de lesión disminuye, la embarazada, podrá llevar una vida diaria más satisfactoria y esto tendrá una traducción directa en el concepto de calidad de vida ${ }^{(20)}$.

Como se ha destacado a lo largo del documento, se realizó un seguimiento del final del embarazo, analizando cómo fue el parto. Si el programa de actividad física por medio del método Pilates ha tenido un efecto positivo en la calidad de movimiento, no menos desfavorecedor ha sido el resultado en cuanto al parto (tabla 3). Destaca, como todos los partos han sido eutósicos, no existiendo ninguna gestante con parto distócico (instrumentado, demasiado lento, etc.), ni por cesárea. Existen estudios que ponen de manifiesto como el ejercicio físico puede ayudar a la embarazada a la hora de dar a luz ${ }^{(4)}$, donde en este caso, el método Pilates puede ser a su vez una buena alternativa. Si la influencia del ejercicio puede explicar este hecho, más aún lo pone de manifiesto en la ausencia de necesidad de episiotomía (tabla 3). Si una embarazada no necesita episiotomía, su recuperación posterior va a ser mucho más acelerada. Tiene lógica pensar que si el programa por medio del método Pilates está diseñado para que exista una parte de tonificación de posturas específicas para embarazadas que involucran fortalecimiento del suelo pélvico, esta pueda ser una consecuencia. En este estudio, no se ha evaluado el suelo pélvico, pero es bien conocido como el incremento en la fuerza y flexibilidad de esta musculatura colabora a que no sea necesaria la episiotomía (31).

En relación a la analgesia epidural en el parto, existe un cincuenta por ciento de la muestra que la usó y el mismo porcentaje que no (tabla 3). En general, un programa de actividad física favorece un mejor control del dolor 
durante el parto ${ }^{(32)}$, y por tanto, la ausencia de necesidad de analgesia. De hecho, esto también se refleja en los centímetros de dilatación de las gestantes. Es cierto, que este estudio posee algunas limitaciones. En primer lugar, que es un estudio piloto, dado que la muestra es muy pequeña, lo que puede haber influido en alguno de los parámetros analizados, aspecto que deberá ser subsanado en el futuro. Por otro lado, la duración del programa se considera correcta, no obstante, se desconoce si una mayor o menor duración e, incluso, frecuencia de sesiones puede influir más o menos positivamente. No obstante, este tipo de investigación, a pesar de su carácter de estudio piloto, pone de manifiesto la necesidad de seguir investigando en esta área de conocimiento ya que los resultados preliminares son positivos y alentadores para una población como las gestantes.

\section{CONCLUSIÓN}

Un programa de actividad física de ocho semanas de duración por medio del método Pilates en mujeres embarazadas, ofrece beneficios en la mejora de la funcionalidad y por lo tanto una disminución del riesgo de lesión. Por otro lado, existe un efecto positivo en el parto, siendo el mismo de carácter eutósico y sin analgesia.

\section{REFERENCIAS}

1. JM. Cea. The effect of gestational weight gain by body mass index on maternal and neonatal outcomes. 2009;31:28-35.

2. G. T-Lea. Seguimiento de un programa de actividad física en el medio acuático para mujeres embarazadas. 2012;11:84-92.

3. White E, Pivarnik J, Pfeiffer K. Resistance Training During Pregnancy and Perinatal Outcomes. Journal of Physical Activity \& Health. 2014;11(6):1141-8.

4. Barakat R, Perales M, Bacchi M, Coteron J, Refoyo I. A Program of Exercise Throughout Pregnancy. Is It Safe to Mother and Newborn? American Journal of Health Promotion. 2014;29(1):2-8.

5. Harris ST, Liu JH, Wilcox S, Moran R, Gallagher A. Exercise During Pregnancy and its Association with Gestational Weight Gain. Maternal and Child Health Journal. 2015;19(3):528-37.

6. Torres-Luque G, Torres-Luque L, García-Chacón S, Villaverde C. Seguimiento de un programa de actividad física en el medio acuático para mujeres embarazadas. Kronos; 2012. 84-92.

7. Aparicio VA, Ocon O, Padilla-Vinuesa C, Soriano-Maldonado A, RomeroGallardo L, Borges-Cosic M, et al. Effects of supervised aerobic and strength training in overweight and grade I obese pregnant women on maternal and foetal health markers: the GESTAFIT randomized controlled trial. Bmc Pregnancy and Childbirth. 2016;16:13.

8. Price BB, Amini SB, Kappeler K. Exercise in Pregnancy: Effect on Fitness and Obstetric Outcomes-A Randomized Trial. Medicine and Science in Sports and Exercise. 2012;44(12):2263-9.

9. Barnes Pea. Resistance training during pregnancy. 2007;29:44-6.

10. Fernández-Arranz M, Lambruschini R, Fernández Arranz J. Manual de Pilates aplicado al embarazo. Madrid: Panamericana; 2016.

11. Ochoteco M, Colella S. Método Pilates Manual teórico-práctico: Ediciones al Margen; 2011. 
12. Mazzarino M, Kerr D, Wajswelner H, Morris ME. Pilates Method for Women's Health: Systematic Review of Randomized Controlled Trials. Archives of Physical Medicine and Rehabilitation. 2015;96(12):2231-42.

13. Serrano-Gómez V, García-García O. Ejercicio físico y Pilates durante el embarazo. Efdeportes. Revista digital.

14. Han IH. Pregnancy and spinal problems. Current Opinion in Obstetrics \& Gynecology. 2010;22(6):477-81.

15. Rostami M, Noormohammadpour P, Mansournia MA, Hantoushzadeh S, Farahbakhsh F, Nourian R, et al. Comparison of the Thickness of Lateral Abdominal Muscles Between Pregnant Women With and Without Low Back Pain. Pm\&R. 2015;7(5):474-8.

16. HC. Oea. Back pain in relation to pregnancy: A 6- year follow-up. 1997;22:294550.

17. S. Mea. Previous history of LBP with work loss is related to lingering deficits in biomechanical, physiological, personal, psychosocial and motor control characteristics. 2003;46:731-46.

18. McGill SM, Andersen JT, Horne AD. Predicting performance and injury resilience from movement quality and fitness scores in a basketball team over 2 years. Journal of Strength and Conditioning Research. 2012;26(7):1731-9.

19. K. Kea. Can serious injury in professional football be predicted by a preseason functional movement screen? 2007;2:147-58.

20. J. Nea. Pilates improves pain, function and quality of life in patients with chronic low back pain: a randomized controlled trial. 2015;29:59.

21. G. Cea. Functional movement screening: the use of fundamental movements as an assessment of function. Part 1. 2014;9:396-406.

22. G. Hea. Agrement of an Evaluation of the Forward-Step-Down Test by Broad Cohort of Clinicians with that of an Expert Panel. 2016;25:227-32.

23. JM. Mea. Reliability and Validity of the Active Straight Leg Raise Test in Posterior Pelvic Pain Since Pregnancy. 2001;23:1167-71.

24. M. F-Aea. Manual de Pilates aplicado al embarazo. 2016.

25. Robinson L. The Body Control. Pilates Pregnancy Book. 2007.

26. M. Fea. Effects of mat Pilates programme on muscular strength and endurance in elderly women. 2012;18:299-307.

27. Brewin D, Naninni A. Women's perspectives on falls and fall prevention during pregnancy. American Journal of Maternal-Child Nursing. 2014;39(5):300-5.

28. Fourie M, Gildenhuys GM, Shaw I, Shaw BS, Toriola AL, Goon DT. Effects of a mat pilates program on flexibility in elderly women. Medicina Dello Sport. 2013;66(4):545-53.

29. Cruz-Ferreira A, Fernandes J, Kuo YL, Bernardo LM, Fernandes O, Laranjo L, et al. Does Pilates-Based Exercise Improve Postural Alignment in Adult Women? Women \& Health. 2013;53(6):597-611.

30. Zakas A. The effects os streching duration on the lower-extremety flexibility of adolescent soccer players. Journal of Body-Work and Movement Therapies; 2005. p. 220-5.

31. Tapullima-Pérez E, Quenaya-Amasifuén K. Técnica de la esferoterapia de pilates en el Programa de Psicoprofilaxis Obstétrica en beneficio del periodo expulsivo en nulíparas. Hosp. Nal. Docente Madre Niño San Bartolomé; 2009.

32. Borreguero-Cardeñosa M. La actividad física durante el embarazo y su influencia en el proceso de parto y en la recuperación posparto. Reduca (Enfermería, Fisioterapia y Podología) Serie Matronas. 2012;4 (5): 1-42.

Referencias totales citadas: 32

Referencias citadas correspondientes a la Rev lb CC Act Fis Dep: 0 